\title{
XIV. Ueber einige Kupfererze und Beiträge zur Kenntniss der Zirkongruppe.
}

\author{
Von
}

S. Stevanovic in Belgrad.

(Mit 7 Textliguren.)

\section{Ueber Stylotyp, Famatinit und Enargit.}

In der mineralogischen Staatssammlung zu Yünchen befinden sich einige Kupfererze von der Grube "Caudalosa Costrovirroyna in Peru. Nähere Untersuchungen ergaben das Vorhandensein folgender Mineralien an den Stufen: Stylotyp, Enargit und ein an Antimon reicher Luzonit ( $\Lambda$ ntimon-Luzonit oder Stibio-Iuzonit). I)ie Hauptmasse und die erste Bildung gehört dem Stylotyp an, dann folgt, immer begleitet von dichtem Quarz, Antimon-Luzonit, und auf diesem sitzen die Krystalle von Enargit. Die letzte Bildung ist Fahlerz und zwar Tennantit, dessen Krystalle oft auf den Enargitkrystallen sitzen. Hie und da kommen Krystalle von Quarz und Eisenkies, wohl auch Kupferkies, aber nur derb, vor.

Stylotyp. Die schwarze, grösstentheils derbe, metallisch glänzende Masse ergab bei den Analysen:

1.

\begin{tabular}{lr} 
Schwefel & 23,20 \\
Antimon & 22,15 \\
Arsen & 6,20 \\
Wismuth & 1,12 \\
Kupfer & 41,50 \\
Silber & 1,40 \\
Eisen & 2,24 \\
Zink & 1,54 \\
Rückstand & 0,34 \\
\cline { 2 - 2 } Summe & 99,69
\end{tabular}

II. 23,20

26,3

4,32

1,12

36,05

1,34

2,76

$3, \mathbf{4 3}$

$\begin{array}{r}1,41 \\ \hline 99,94\end{array}$
III.

25,75

16,86

6,28

0,73

43,60

1,44

3,98

0,80

0,41
99,85 
Atomverhältnisse:

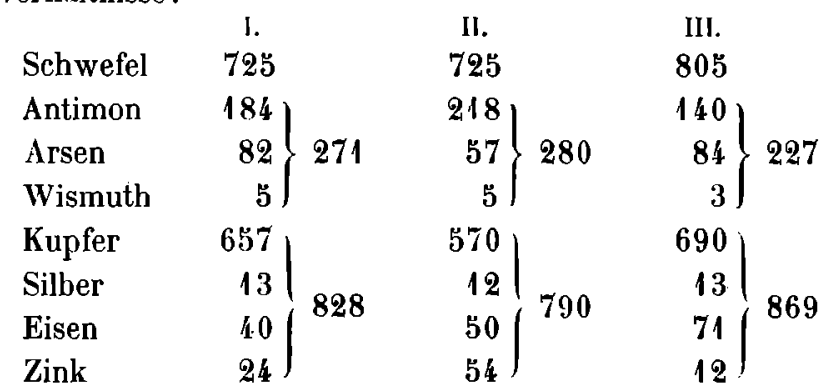

Also

\begin{tabular}{|c|c|c|c|c|}
\hline & Schwefe & : Antimon + & & Metall \\
\hline I. & 2,67 & 1 & & 2,94 \\
\hline II. & 2,68 & 1 & & 2,88 \\
\hline n. & $3, \breve{34}$ & 1 & & 3,82 \\
\hline
\end{tabular}

Obwohl das Material sehr homogen aussieht, zcigen doch die Analysen, dass dies nicht der Fall ist. Ije crsten zwci Analysen stimmen dem $\Lambda$ tomverhällnisse nach gut überein, aber eine einfache Formel daraus abzulciten ist nicht möglich. Deshalb wurde noch eine Analyse (III) von Krystallstücken und deren nächster Umgebung ausgeführt. Während die zwei ersten zuviel Antimon und Arsen für ein normales Sulfosalz zeigten, ergab die dritte, gerade umgekehrt, zu wenig von jenen Elementen. Nimmt man jedoch den: Mittelwerth aus den drei $\Lambda$ nalysen, so ergiebt sich ziemlich genau das Verhältniss Schwefel : $\Lambda$ ntimon- $\Lambda$ rsen : Metall wie $3: 1: 3$. Ebenso stimmen diese Analysen noch am besten mit der des von Scharizer ${ }^{1}$ ) beschriebenen Falkenhaynit von Joachimsthal überein, der ein normales Sulfosalz von der Formel $Q R_{3} S_{3}$ ist, wobei $Q=$ Arsen und Antimon, und $R=$ Kupfer, Silber, Eisen und Zink ist. Also darf man es als ziemlich sicher annehmen, dass wir hier mit einem Minerale von der chemischen Formel $(S b, A s) S_{3}\left(C u, A g, \frac{Z n}{2}, \frac{H e}{2}\right)$ zu thun haben. Ein Fahlerz ist schon von vornherein ausgeschlossen, weil die Farbe etwas dunkler ist, und weil diese Erze sich viel leichter mit Säuren aufschliessen lassen, obwohl sie in anderen Merkmalen mit Fahlerzen viele Aehnlichkeiten haben. Auch die krystallographische Untersuchung spricht entschieden gegen Fahlerz. Nur ist die Frage, was diesem Erze beigemengt ist. Scharizer musste, um obige Formel zu construiren, von seiner Analyse neben $\mathrm{SiO}_{2}$ und magnesiahaltigem Eisenspath auch etwas mehr als $3 \%$ Kupferkies abziehen. Wenn ich von meiner III. Analyse 10,84\% Kupferkies abziche und den Rest auf 100 umrechne, so bekomme ich genau obige Formel (s. unten). Was 22, 85 .

1) Jahrb. d. k. k. geolog. Reichsanst. Wien 1890, 40, 433. Ref. diese Zeitschr. 
aber dem Materiale beigemengt ist, von welchem die I. und II. Analyse gemacht wurden, kann man nicht mit Sicherheit sagen. Allerdings muss es ein Mineral sein, welches an Arsen und Antimon reicher ist, vielleicht Arsenkies, aber dieser ist nicht an der Stufe beobachtet worden.

Dieses Erz stimmt äusserlich vollkommen mit dem von v. Kobell beschriebenen Stylotyp überein 1), dessen Original die mineralogische Staatssammlung zu München besitzt und welches von der Grube Copiapó in Chile stammt. Kobell nahm an, dass er in seiner Analyse Verluste an Schwefel hatte, und stellte daher die schon erwähnte Formel auf, nur fand er mehr Silber und Eisen. Ich untersuehte dieses Erz noch einmal chemisch und fand, dass es zwar der obigen Formel sehr nahe steht, aber dass es ebenso wenig homogen ist, wie jenes von Grube Caudalosa in Peru.

Folgende Tabelle soll die Bezichungen zwischen diesen drei Erzen veranschaulichen.
A.
B.
C.

Stylotyp von Copiapo. Stylotyp von Caudalos. Falkenhaynit. Kobell: Stevanović: Stevanovid. Scharizer:

\begin{tabular}{|c|c|c|c|c|}
\hline \multirow[b]{2}{*}{ Schwefel } & \multirow[b]{2}{*}{21,30} & \multicolumn{3}{|c|}{ Naclı Abz. v. 10,84 $C u S_{2} F_{c}$} \\
\hline & & 23,12 & 24,55 & 25,76 \\
\hline Antimon & 30,53 & 28,58 & 18,99 & 24,30 \\
\hline Arsen & - & - & 7,07 & 5,02 \\
\hline Wismuth & - & - & 0,54 & 0,34 \\
\hline Kupfer & 28,00 & 30,87 & 45,84 & 39,77 \\
\hline Silber & 8,30 & 10,43 & 1,62 & - \\
\hline Eisen & 7,00 & 6,27 & - & 2,82 \\
\hline Zink & Spur & Spur & 0,90 & 1,99 \\
\hline Summe & 98,13 & 99,27 & 99,51 & 100,00 \\
\hline Spec. Gew. & 4,79 & 5,18 & 4,77 & 4,83 \\
\hline
\end{tabular}

Atomverhältnisse nach meinen Analysen:

A.

Copiapo:

Schwefel

Antimon

Arsen

Wismuth

Kupfer

Silber

lïisen

Zink
722

237

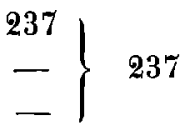

487

97

112
-
B.

Caudalosa :

\section{7}

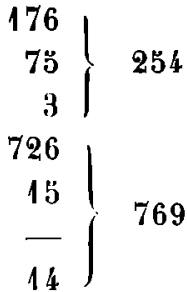

1) Sitzungsber. d. Akad. d. Wiss. in Miinchen $1865,163$. 
Also:
$S \quad: S b, A s:$ Metall :
A. $3,05: 1: 3,41$
B. $3,02: 1: 3,02$

Aus obigen Zahlen geht hervor, dass der Falkenhaynit mit dem Stylotyp identische Formel besitzt und sich nur dadurch von diesem unterscheidet, dass er weniger Silber und Eisen enthält und dass etwas Antimon durch Arsen ersetzt ist. Dem Stylotyp von Caudalosa steht er noch näher. Die gemeinsame Formel dieser Mineralien künnen wir so darstellen: $(S b, A s, B i) S_{3}\left(C u, A g, \frac{Z n}{2}, \frac{F e}{2}\right)_{3}$.

Es sei noch erwähnt, dass das specifische Gewicht 5,18 für Stylotyp besser in die Reihe passt, als 4,79.

Krystallographische Untersuchungen des Stylotyp. Der Stylotyp von Copiapó zeigt zwar grosse, aber schon zersetzte Krystalle, welche mit einer rauhen Rinde umhüllt und deshalb unmessbar sind. Diese Krystalle sind lange, fast rechtwinkelige Prismen, welche wieder so gruppirt sind, dass sie zu einander im Winkel von circa $90^{\circ}$ stehen und ein gestricktes federförmiges Aussehen haben. Alles das veranlasste Kobell, den Stylotyp für »wahrscheinlich rhombisch « zu halten. Scharizer stellte seinen Falkenhaynit in die sWittichenitgruppe «. Die nähere Beobachtung des Kobell'schen Stylotyp zeigte, neben rechtwinkeligen Prismen, noch einige Krystalle von anderem Habitus. Zwei solche wurden isolirt, von der rauhen lRinde mechanisch befreit, mit Schmirgel und Hirschleder polirt und gemessen. Mit IIülfe dieser Messungen konnte ich wenigstens eine angenäherte Bestimmung der Krystallform erhalten und diese ergänzen durch die Messungen, die ich an Krystallfragmenten des Stylotyp von Caudalosa ausgeführt hatte. Es erwies sich, dass Stylotyp dem Xantokon und der Feuerblende (Pyrostilpnit) isomorph ist.

Angenommen $\beta=90^{\circ}$ (bei Xantokon und Feuerblende steht $\beta 90^{\circ}$ sehr nahe) erhielt ich für die $\Lambda$ xenverhältnisse:

$$
a: b: c=1,9202: 1: 1,0355 .
$$

Es wurden folgende Formen beobachtet: $a\{100\}, m\{110\}, n\{210\}$, $u\{310\}, x\{111\}, r\{101\}, q\{313\}, o\{311\}, s\{401\}, t\{302\}, y\{332\}, d\{032\}$.

$\begin{array}{lcc} & \text { Beobachtet: } & \text { Bercchnet: } \\ a: \mu=(100):(310) & =31035^{\prime}-32^{2} 12^{\prime} & 32027^{\prime} \\ a: n=(100):(210) & * 43^{0} 50^{\prime} & - \\ a: m=(100):(110) & 61030^{\prime}-62030^{\prime} & 6230 \\ a: s=(100):(401) & 23 \quad 0-250 & 2452 \\ a: t=(100):(302) & 5200^{\prime} & 51 \quad 0 \\ 11: o=(210):(311) & * 2740 & - \\ a: o=(100):(311) & 4440 & 4341\end{array}$




$\begin{array}{lcc} & \text { Beobachtet: } & \text { Berechnet: } \\ m: y=(110):(332) & =30^{\circ} 30^{\prime} & 29042^{\prime} \\ m: x=(1 T 0):(1 T 1) & 4424 & 4425 \\ x: r=(111):(101) & 4355 & 4221 \\ b: d=(010):(032) & 3240 & 3345 \\ d: x=(032):(111) & 2440 & 2550\end{array}$

$b(010)$ ist sehr unvollkommen, und man kann nicht sagen, ob sie natürliche Fläche ist oder gespalten. Die Fläche $q(313)$ ist durch die Zonen bestimmt worden. Alle Flichen sind stark gestreift und geben schlechte Reflexe, deshalb dürfen die Messungen nur als annähernd betrachtet werden. Eins ist jedoch sicher, nämlich dass Stylotyp mit Xantokon und Feuerblende isomorph ist, wie es schon vermuthet worden war (vergl. auch Groth, Tabellar. Uebers. d. Miner. S. 35).

Stellen wir diese drei Mineralien neben einander, so haben wir:

Xantokon $A s S_{3} A g_{3} \quad a: b: c=1,9187: 1: 1,0152 ; \beta=91^{\circ} 13^{\prime}$.

Spec. Gew. 5,45 .

Feuerblende $S b S_{3} A g_{3} \quad a: b: c=1,9465: 1: 1,0973 ; \quad \beta=90^{0} \mathrm{ca}$.

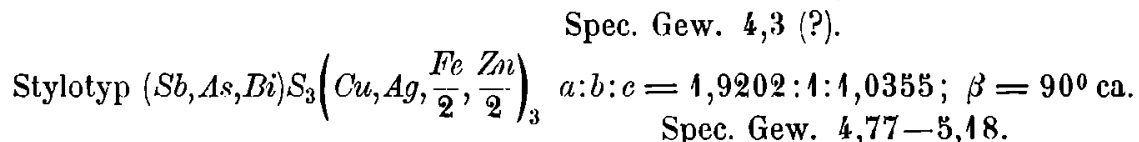

Ueber den Habitus der Krystalle sei noch erwähnt, dass die Zahl der beschriebenen Krystalle weit übertroften ist von jenen, welche fast rechtwinkelige Prismen besitzen und deren Winkel IIessenberg ${ }^{1)} \mathbf{z u} 921_{2}^{0}$ schätzte. Obwohl die Flïchen $(210):(\overline{2} 10)$ den Winkel von $92020^{\prime}$ bilden, also dem obigen sehr nahe, so ist doch wenig wahrscheinlich, dass die Prismen von diesen Flächen gebildet sind. Viel wahrscheinlicher ist, dass wir hier beide Pinakoïde, (100) und (010), haben, folglich den Winkel von $90^{\circ}$. Unter dieser Annahme wäre auch dic Vermuthung von Kobell bestätigt, dass die Prismen in Zwillingsstellung stehen. Nehmen wir $x(111)$ als Zwillingsebene, so bilden die Kanten dieser Prismen Winkel von 880 $50^{\prime}$ (zweimal 44025'), also auch einen Winkel, welcher $90^{\circ}$ sehr nahe steht. Die Flächen (100) und (100) müssen dann Winkel von $38^{\circ} 30^{\prime}$ umschliessen, und in der That beträgt dieser, an einem Kittabdrucke mit dem Contactgoniometer gemessen, $38^{0}$.

Antimon-Luzonit. A. Stelzner ${ }^{2}$ ) nannte Famatinit einen Kies von dem Famatina-Gebirge in Argentinien, wclcher die chemische Zusammensetzung des Enargit hatte, in welchem aber der grüsste Theil des Arsens

1) Siehe die $\Lambda$ rbeit von Kobell.

2. Mineral. Mith. 1873, 240. 
durch Antimon ersetzt ist. Ein Mineral, welches äusserlich dem obigen vollkommen gleich ist, aus den Kupfererzgängen von Mancayan auf der Insel Luzon, welches aber genau die Zusammensetzung des Enargit hat, wurde von Weisbach ${ }^{1}$ ) untersucht und Luzonit genannt. Eine Stufe von Cerro de Pasco in Peru untersuchte A. Frenzel2) und fand Arsen und Antimon fast im Gleichgewicht; sonst stimmt auch dieses Erz vollkommen mit Famalinit und Luzonit überein, nümlich alle drei haben rüthlichgraue Farbe, zeigen keine Spur von Spaltbarkeit und kommen immer dicht und eingesprengt vor.

In dem Stylotyp von der Grube Caudalosa ist ein Mineral von röthicher Farbe eingesprengt, ohne Spuren von Spaltba rkeit. Die chemische Untersuchung ergab hier dieselbe Zusammensetzung, welche Frenzel bei dem Kiese von Cerro de Pasco fand, nämlich:

I.

lirenzel.

$\begin{array}{lr}\text { Schwefel } & 30,45 \\ \text { Antimon } & 12,74 \\ \text { Arsen } & \mathbf{8 , 8 8} \\ \text { Kupfer } & 47,93 \\ \text { Eisen } & - \\ \text { Rückstand } & - \\ \text { Summe } & \mathbf{1 0 0 , 0 0}\end{array}$

Spec. Gew. 4,39
II. Stevanovic.

$\begin{array}{ccc}\text { a. } & \text { b. } & \text { Mittel: } \\ 31,04 & 30,99 & 31,01\end{array}$

$12,13 \quad 13,36 \quad 12,74$

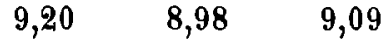

$45,50 \quad 45,36 \quad 45,43$

$0,65 \quad 0,70 \quad 0,67$

\begin{tabular}{crrr}
0,80 & 0,51 & 0,65 \\
\hline 99,32 & 99,90 & 99,59
\end{tabular}

4,47 bei $15^{0}$

Atomverhältnisse nach den Mittelwerthen:

\begin{tabular}{|c|c|c|}
\hline Schwefel & & 969 \\
\hline Antimon & 106 & 227 \\
\hline Arsen & $121\}$ & \\
\hline Kupfer & 719 & 733 \\
\hline
\end{tabular}

Es ist leicht ersichtlich, dass etwas Eisenkies beigemengt ist, was unvermeidlich ist. Abgezogen 1,44\% $\mathrm{Fe} S_{2}$ und auf 100 umgerechnet (siehe unten) resultirt das Verhältniss $(A s, S b): S: C u=1: 4,11: 3,13$, was unbedingt der Formel $(A s, S b) S_{4} C u_{3}$ entspricht.

Die folgende Tabelle zeigt die Bezichungen zwischen diesen Kiesen.

1) Mineral. Mitth. 1874, 257.

2) N. Jahrb. f. Min. etc. $1874,685$. 


\section{Luzonit: Antimon-Luzonit: Famatinit 1):}

\begin{tabular}{|c|c|c|c|}
\hline Schwefel & 33,14 & 34,02 & 29,40 \\
\hline Arsen & 16,52 & 9,32 & 3,84 \\
\hline Antimon & 2,15 & 13,07 & 21,44 \\
\hline Kupfer & 47,51 & 46,59 & 44,72 \\
\hline Eisen & 0,93 & - & 0,67 \\
\hline \multirow[t]{2}{*}{ Zink } & - & - & 0,59 \\
\hline & 100,24 & 100,00 & 100,66 \\
\hline Spec. Gew. & 4,24 & 4,47 & 4,57 \\
\hline
\end{tabular}

Ueber die Krystallform dicser Erze ist noch nichts bekannt. Frenzel hielt sie für wahrscheinlich monoklin. Zufälligerweise hat vom Rath ${ }^{2}$ ) Krystalle von Enargit, welche ihm Stelzner überreichte, anstatt des Famatinit gemessen, und crklärte, Famatinit sei mit Enargit isomorph. Spitere Untersuchungen von Klockmann ${ }^{3}$ ) an Luzonit von Sierra de Famatina zeigten, dass dies nicht der Fall war, sondern dass wir es hier mit einem dimorphen Körper zu thun haben. Einerseits sind Luzonit und Famatinit isomorph, wie schon Stelzner und Weisbach vermuthet haben, was auch der dazwischen stehende Antimon-Luzonit als isomorphe Mischung beider bestätigt, während andererseits der chemisch dem Luzonit entsprechende Enargit noch isolirt steht.

Enargit. Nach der ausführlichen Arbeit über die Krystallformen des Enargits von L. S. Spencer'y kann ich mich beschränken nur auf das, was ich an dem Enargit beobachtet habe, ohne die Geschichte dieses Minerals zu wiederholen. Wie schon erwähnt ist, sitzen auf dem Stylotyp oder Antimon-Luzonit von Caudalosa in Hohlräumen kleine, glïnzende, sehr liichenreiche Krystalle von Enargit. Es wurden 20 isolirt und gemessen. Im Ganzen zeigten sie 27 Formen, von denen zwölf zum ersten Male beobachtet und zwei bis jetzt zweifelhafte bestätigt wurden, nämlich: $a\{100\}$, $b\{010\}, c\{001\}, y\{610\}, S\{410\}, f\{520\}, x\{320\}, \quad T\{430\}, F\{980\}$, $N\{230\}, h\{120\}, P\{250\}, l\{130\}, L\{270\}, R\{140\}, Q\{150\}, D\{160\}$, $e\{403\}, G\{041\}, J\{232\}, s\{011\}, M\{201\}, k\{101\}, n\{102\}, \delta\{031\}$, $B\{205\}, H\{061\}$. Alle mit grossen Buchstaben bezeichneten Formen ausser $M$ und $N$ sind neu.

Die Tabelle zeigt die beobachteten und berechneten Werthe, welche mit einander vorzüglich übereinstimmen. Die Rechnung bezieht sich auf

1) Mittelwerth von vier Analysen von Sievert, Mineral. Mitth. 1873, S. 240.

2) Sitzungsber. d. Niederrhein. Gesellsch. für Natur- u. Heilkunde 1878, S. 179. A usz. diese Zeitschr. 4, 426.

3) Diese Zeitschr. 19, 272.

4) The Mineralogical Magazine 180:, 11, Nr. 50, 11, 69-79. read $\Lambda$ pril. Ref. diese Zeilschr. 1997, 28, 210. 
mein Axenverhältniss $a: b: c=0,8665: 1: 0,8299$, welches von jenem von Dauber $a: b: c=0,869 / 4: 1: 0,8308$ wenig verschieden ist.

\begin{tabular}{|c|c|c|c|c|c|}
\hline & Beob. & & Berechnet: & Grenzwerthe & \\
\hline$n: m=(110):(1 \bar{T} 0)=$ & $* 81059^{\prime}$ & 25 & - & $81^{0} 46^{\prime}-8201$ & \\
\hline$: \delta=(001):(051)$ & *76 27 & 10 & 一 & $76 \quad 14-76$ & 49 \\
\hline$: y=(100):(610)$ & 749 & 1 & $8^{0} 12^{\prime}$ & - & \\
\hline$: S=(100):(410)$ & 1226 & 3 & 1213 & $1115-131$ & $1 \ddot{3}$ \\
\hline$: f=(100):(520)$ & 1931 & 8 & 197 & $190-20$ & 2 \\
\hline$: d=(100):(210)$ & 2328 & 11 & $2324.40^{\prime \prime}$ & $22 \quad 0-25$ & \\
\hline$: x=(100):(320)$ & $\begin{array}{ll}30 & 39\end{array}$ & 3 & $\begin{array}{lll}30 & 0 & 43\end{array}$ & $3035-30$ & 4.5 \\
\hline$: T=(100):(430)$ & 335 & 2 & 331 & $3248-332$ & 22 \\
\hline$: F=(100):(980)$ & $3750 \frac{1}{2}$ & 9 & 3736 & $3655-385$ & 55 \\
\hline$a: N=(100):(230)$ & 3135 & 4 & 5225 & $5045-525$ & 59 \\
\hline$a: h=(100):(120)$ & $60 \quad 0$ & 10 & $60 \quad 0 \quad 14$ & $5953-601$ & 15 \\
\hline$a: P=(100):(250)$ & $65 \quad 25$ & 7 & $\begin{array}{lll}65 & 13\end{array}$ & $64 \quad 0-66$ & 48 \\
\hline$a: l=(100):(130)$ & 6835 & 10 & $6857: \frac{1}{2}$ & $68 \quad 22-69$ & \\
\hline$a: L=(100):(270)$ & 7130 & 6 & 7143 & $6956-713$ & 34 \\
\hline$a: R=(100):(140)$ & 7318 & $7^{\circ}$ & 7354 & $7230-74$ & 47 \\
\hline$a: Q=(100):(150)$ & 7720 & 1 & 770 & - & \\
\hline$a: D=(100):(160)$ & 7914 & 1 & 797 & - & \\
\hline$c: e=(001):(403)$ & 5145 & 1 & 5156 & - & \\
\hline$c: G=(001):(041)$ & 728 & 1 & 7314 & - & \\
\hline$c: J=(001):(232)$ & 5725 & 2 & 5734. & $5725-572$ & 25 \\
\hline$J: J=(232):(\overline{2} 32)$ & 620 & 1 & 6158 & - & \\
\hline$c: s=(001):(011)$ & 3938 & 3 & 3941 & $39 \quad 16-40$ & \\
\hline$c: M=(001):(201)$ & $62 \quad 15$ & 1 & 6296 & - & \\
\hline$c: k=(001):(101)$ & $43 \quad 40$ & 1 & 4346 & - & \\
\hline$c: n=(001):(102)$ & 2539 & 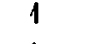 & 2535 & - & \\
\hline$c: B=(001):(205)$ & 2033 & 1 & 2058 & - & \\
\hline$: H=(001):(061)$ & 7854 & 1 & 7839 & - & \\
\hline
\end{tabular}

Damit sind am Enargit im Ganzen ih Formen beobachtet. Zur Be. rechnung der Axe $c$ wurde $\{051\}$ genommen, weil diese Form gewühnlich vorkommt und gute Reflexe liefert.

Die neuen Formen sind gewöhnlich mit sehr schmalen Flïchen ausgebildet, aber diese sind glänzend, und wenn sie etwas grösser sind, treten sie an guter Ausbildung gegen die anderen Flïchen nicht zurück. Manchmal sind alle fast im Glcichgewichte an einem Krystalle entwickelt, was demselben ein gerundetes Aussehen gicbt. Die Form $x\{320\}$ wurde dreimal beobachtet, aber immer war sie sehr schmal; schon Spencer bemerkte, dass sie vorhanden sei, konnte sie aber nicht messen. Die Form \{232\} 
ist sehr oft vorhanden, aber fast immer rauh und unmessbar, nur an einem Krystalle war sie glatt und gab gute Reflexe. Zweifelhaft bleiben die Formen $F\{980\}$ und $G\{041\}$. Die erste, obwohl neunmal beobachtet, weil man nicht sagen kann, ob sie die Indices (980) oder (10.9.0) oder (870) hat. Die zweite ist nur einmal in der Zone mit $\{011\}$ beobachtet, war sehr gross, aber der berechnete Werth stimmt besser mit den Indices (0.15.4), als mit (041). Die Form $\{403\}$, welche von Dauber beobachtet wurde, aber welche Goldschmidt für zweifelhaft erklärte, wie auch die von Spencer beobachteten Formen $\{520\}$ und $\{610\}$ wurden bestitigt.

Die Krystalle sind von zweicrlei Typus: tafelförmig nach (100) oder säulenförmig-pseudohexagonal, wobei die herrschenden Formen $a\{100\}$ und $h\{120\}$ sind. Merkwürdig ist, dass die Formen $\{051\}$ niemals, und die

Fig. 1.

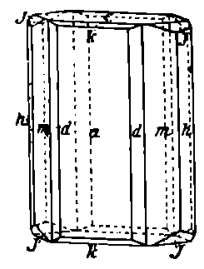

Fig. 2.

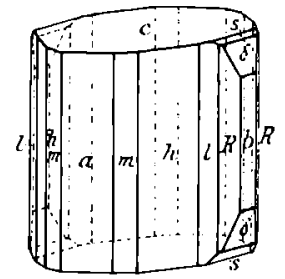

Fig. 3.

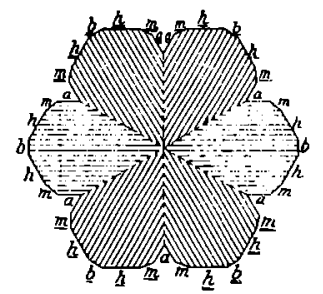

$\{120\}$ und $\{010\}$ sehr selten und untergeordnet bei dem ersten Typus beobachtet sind (Fig. 1). Umgekchrt sind $k\{101\}, d\{210\}$ sehr selten, $J\{232\}$ gar nie an dem zweiten Typus beobachtet, wo immer die herrschende Form $h\{120\}$ ist (Fig. 2). Im Allgemeinen sind Endflächen selten und gewöhnlich matt.

Drillinge und $Z$ willinge sind hïufig, aber niemals beim ersten Typus. Einige Drillinge (s. Fig. 3, Projeclion auf $(001)$ ) sind von ausgezeichneter Ausbildung. Aus meinen Messungen konnte ich keinen Grund sehen, die Fläche (320), welche vom Rath als Zwillingsebene angenommen hat und welche auch Spencer als solche bevorzugte, statt (120) als Zwillingsebene anzunehmen. Ja sogar wahrscheinlicher ist, dass $h(120)$, cine häufigere Form, welche den Krystallen den hexagonalen IIabitus verleiht, Zwillingsebene ist, um so mehr, als die Zwillinge nur beim zweilen Typus beobachtet werden. Wenn die Form $b\{010\}$ bei den Drillingen etwas besser entwickelt ist, dann haben diese ein vollstiindig hexagonales Aussehen. In solchen Fällen verrathen nur die Strcifensysteme auf der Basis, welche bei den einzelnen Individuen parạllel mit der Makrodiagonale laufen, die Drillingsbildung.

Chemische Untersuchung des Enargits. Spaltungsstücke und schlechte krystalle wurden fïr die Analyse verwendel, welche ergab: 


$\begin{array}{lcc} & & \text { Atomverhältnisse } \\ \text { Schwefel } & 32,42 & 1015=4,01 \\ \text { Arsen } & 19,08 & 253=1 \\ \text { Kupfer } & 48,53 & 769=3,04\end{array}$

Also $\Lambda$ rsen : Schwefel : Kupfer wie $1: 4: 3$, folglich die Formel $\mathrm{AsS}_{4} \mathrm{Cu}_{3}$ eines typischen antimonfreien Enargits.

Das specilische Gewicht wurde an einer Partie zu 4,4,40, an einer anderen zu 4,488 bei $14^{\circ}$ gefunden, im Mittel 4,464 (frühere Angaben: 4,36 $-4,47)$.

Auf den Krystallen von Enargit, innig mit diesen verwachsen, sitzen manchmal kleine, metallisch glänzende kubische Krystalle, an denen die Formen $\{110\}$ herrschend, $\{211\}$ und $\{100\}$ beobachtet wurden. Aus Mangel an Material wurde nur eine qualitative Analyse ausgeführt, welche ergab: Arsen, Schwefel und Kupfer, also ein reiner Tennantit.

Methode der Analysen. An möglichst reinem Material wurde zuerst das specifisclie Gewicht bestimmt. Etwa 0,6 bis $1 \mathrm{~g}$ Substanz wurde bei $95^{0}$ getrocknet und nach dem schon bekannten Verfahren im Pyknometer bchandelt. Für jede Bestimmung lagen wenigstens sechs Beobachtungen vor. Zuletzt der Sicherheit wegen, wo nur eine Portion zur Verfïgun! war, ist die Substanz getrocknet und noch einmal in der Luft gewogen worden. Die angegebenen Werthe sind Mittel von diesen Beobachtungen.

Dieselhen Stücke wurden für die $\Lambda$ nalysen verwendet. Das fein gepulverte, getrocknete Material wurde nach den Methoden von Jannasch 1) anstall im Chlor- im Bromstrome zersetzt. Die Güchtigen Bromide des Arsens, Antimons und Wismuths wurden von getrockneter $\mathrm{CO}_{2}$ in eine mit Salzsäure, Weinsäıre und etwas Salpetersäure gefüllte Vorlage getrieben. Aus der hier erhaltenen Lösung wurde zuerst Schwefelsäure mit Baryumchlorid gefallt und abfiltrirt, dann uberschüssiges Baryumchlorid mit verdünnter Schwefelsäure weggeschafrt, in der Lösung Magnesium als Arsenal niedergeschlagen und als $A s_{2} \mathrm{Mg}_{2} \mathrm{O}_{\bar{\tau}}$ gewogen. Nach dem Abdampfen des Ammoniak wurden $\Lambda$ ntimon und Wismuth durch Schwefelwasserstoff gefillt und mit Hülle von Schwefelammonium getrennt. Wismuth wurde als $\mathrm{Bi}_{2} \mathrm{O}_{3}$ und Antimon als $S b_{2} S_{3}$ gewogen. Die nicht flüchtigen Bromide des kupfers, Eisens, Zinks und Silbers wurden nit lrischem Chlorwasser behandelt, um in Chloride umgewandelt zu werden, vom Riuckstande und Silberchlorid abfiltrirt und Kupfer als $C u_{2} S$ oder Rhodanür, Eisen und 7ink als Oxyde gewogen. Lm das Silber vom Rückstande zu trennen, wurde dieses mit Ammoniak behandelt, das Silber von Neuem in Chlorid umgewandelt und als $\mathrm{AgCl}$ gewogen. Unlösliches in Ammoniak ist bei den Analysen als Rückstand oder Gangart angegeben.

Immer wenn mehr Material zur Verfügung war, wurde der Schwefel in einer anderen Portion bestimmt, weil bei Beltandlung im Bromstrome sich immer etwas Schwefel als Element abscheidet, und dann ist es schwer ihn wieder in Lösung zu bringen. Allein wo wenig Material war, ist der Schwefel aus der Vorlage, nach genügender Behandlung mit Brom, in Lösung gebracht und mit Baryumchlorid gefällt worlen.

1) Praktischer Leitfaden d. Gewichtsanalyse, Leipzig 1897. 


\section{Künstlicher Domeykit.}

Der Domeykit wurde zuerst bei San Antonio in Copiapó (Chile) gefunden und von Zincken ${ }^{1)}$ Arsenikkupfer genannt. Später wurde er noch an einigen Orten Amerikas und Europas gefunden und von llausmann Ionneykit genannt. Er kommt immer in nierenförmigen, traubigen Massen und eingesprengten Partien vor, sehr selten homogen. Frisch ist er metallglïnzend, von zinnweisser oder stahlgrauer Farbe, aber an der Luft wird er gleich matt und angelaufen. Dichte 6,7-7,8.

In neuerer Zeit beschäftigte sich G. $\Lambda$. Koenig ${ }^{2}$ ) mit dem Domeykit von der Halbinsel Keweenaw und fand einen nickel- und kobalthaltigen Domeykit, welchen cr Mohawkit nannte; einen anderen, welcher etwas Anlimon in Vertretung des Arsen zeigte, nannte er Stibiodomeykit. Dic Dichte des Mohawkit fand er 8,07, des Domeykit 7,948.

Bei dieser Gelegenheit stellte Koenig einige Kupferarsenide künstlich dar, erhielt aber keine Krystalle (sichc l. c.). Spätere Versuche waren jedoch erfolgreicher, er erhielt Krystalle von Domeykit.

Die Krystalle des künstlichen Domeykit, welche Herr Prof. Koenig an das mineralogische Institut in München sandte, sind kleine sechsseitige Täfelchen, manchmal so dünn, dass sic biegsam sind, von stahlgrauer Farbe metallischem Glanze; aber an der Luft nehmen sie einc braune Anlauffarbe an. Es wurden dreizehn Krystalle gemessen. Die Messungen erwiesen eine anscheinend vollständige hexagonale Symmetrie. Die Prismenund Pyramidenwinkel unterscheiden sich nur um wenige Minuten von einander. Darstellung von Aetzfiguren ist nicht gelungen, aber es wurde doch ein Merkmal beobachtet, welches für die rhombische Symmetrie spricht, nämlich die Krystalle spalten nur in einer Richtung. Die Krystalle sind zu klein und nie ringsum ausgebildet, noch dazu sind die einander zur hexagonalen Symmetrie ergänzenden Formen immer da, so dass kein Mittel geboten war, um die Prismenflächen von Pinakoiden, resp. Pyramiden von Domen, zu unterscheiden. Doch muss der Unterschied sehr klein sein, weil die berechneten Werthe gut mit den beobachteten stimmen.

$$
a: b: c=0,5771: 1: 1,0206 .
$$

Combination: $c\{001\}, m\{110\}, b\{010\}, p\{111\}$, $d\{021\}, v\{112\}, g\{011\}, \approx\{113\}, e\{023\}, r\{043\}$, $t\{0.1\}, q\{0.5 .12\}$. Der Habitus der Krystalle, wie die Combination selbst, sind denjenigen des Kupferglanzes so ähnlich, dass ich für zweckmüssig finde, die Winkelwerthe dieser zwei Mineralien neben ein-

Fig. 4.

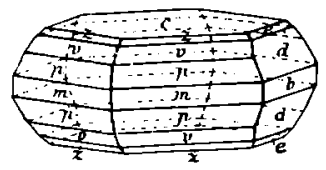
ander zu stellen. 
Domeykit:

Kupferglanz:

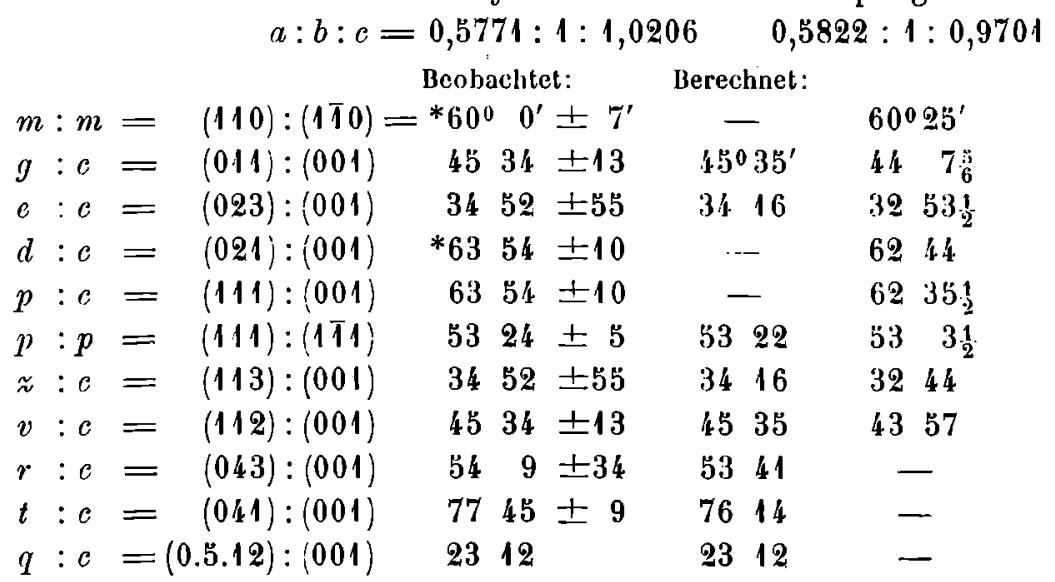

P'seudohexagonalen Charakter geben dem Domeykit hauptsächlich die Formen $\{111\}$ und $\{021\},\{113\}$ und $\{023\}$. Die l'orm $\{112\}$ ist immer vorhanden und besilzt nach $\{111\}$ die besten Flächen, dagegen wurde $\{011\}$ nur zweimal durch die Lupe beobachtet. Die Formen $\{043\}$ und $\{041\}$ sind selten und geben immer schlechte Reflexe. Die Form \{0.5.12\} ist nur einmal durch die Lupe gesehen worden und deswegen zweifelhaft.

Nehmen wir aber die zwar zwölf Mal beobachtete, aber immer untergeordnete Fläche (023) als primäres Doma, d. h. $\{011\}$, so erhalten wir: $a: b: c=0,5771: 1: 0,6802$, und die Combination: $c\{001\}, m\{110\}$, $b\{010\}, p\{332\}, d\{031\}, v\{331\}, g\{032\}, z\{112\}, e\{011\}, r\{021\}, t\{061\}$ und $q\{058\}$. Vergleichen wir dies mit Diskrasit, so erhalten wir:

Domeykit:

$$
a: b: c=0,5771: 1: 0,6802
$$

$m: m=(110):(1 \overline{1} 0)=60^{\circ} 0^{\prime}$

$e: c=(011):(001) \quad 3416$

$\approx: c=(112):(001) \quad 3416$

$r: c=(021):(001)$

$53 \quad 41$

Diskrasit:

$0,5775: 1: 0,6718$
$60^{0} \quad 1^{\prime}$
$33 \quad 53 \frac{1}{2}$
$33 \quad 53$
$53 \quad 20 \frac{1}{2}$

Also sind die Werthe sehr nahe, aber die Combination weniger ähnlich. Diese zwei Körper könnten also als isomorph betrachtet werden, was für die Formel $A g_{3} S b$ für den Diskrasit sprechen würde.

Spaltbarkeit nach (100) deutlich. An einer Partie von 0,1/296 g wurde das specifische Gewicht bestimmt, so genau wie mit so wenig Material möglich war, und $\mathrm{zu} 7,92-8,10$ bei $14^{\circ} \mathrm{C}$. gefunden (übereinstimmend mit der Bestimmung 7,94 von Koenig!. Iiskrasit hat das specifische Gewicht $9,4-9,9$.

Herr Prof. Koenig wird die Frage der Isomorphie dieser Körper weiter verfolgen und über die Darstellung derselben berichten. 


\section{Beiträge zur lienntniss der Mineralien der Zirkongruppe.}

Obwohl der Zirkon ein sehr verbreitetes Mineral ist und vielfach untersucht wurde, so blieben doch noch manche Verhältnisse desselben unaufgeklärt: so ist z. B. nach den Ansichten von J. W. Retgers') und 11. Traube2) seine Isomorphie mit Rutil und Kassiterit als zweifelhaft zu belrachten. Ausserdem variiren manche seiner physikalischen Eigenschaften sehr. Besonders ist dies der Fall bei dem specifischen Gewichte, eine Lirscleinung, welche schon lange bekannt, aber noch nicht genügend erklärt ist. In Lehrbüchern werden die Grenzen 4,5-4,7 angegeben, aber thatsüchlich sind diese Girenzen noch weiler, von etwa 4,0-4,7, was bei keinem anderen Minerale vorkommt. Ferner ist auch bekannt, dass die Zirkone, welche niedrigeres specifisches Gewicht haben, dieses nach dem Glühen zu erhöhen fflegen. Jiese Erscheinung wurde von L. Svanberg ${ }^{3}$ ), Henneberg ${ }^{4}$ ), A. Il. Church ${ }^{5}$ ) u. A. Jeobachtet ${ }^{6}$ ); besonders wichtig ist in dieser Ilinsicht die Arbeit von Church, aus welcher hervorgeht, dass die Zirkone nach ihren specifischen Gewichten in drei Kategorien getheilt werden können, nüınlich: aj solche vom specifischen Gewichte 4,02 (die localitït ist nicht angegeben); b) solche mit 4,705 (Zirkon von Mudgee, New South Wales; wolche ihr specilisches Gewicht durch Glühen nicht veründern, und c) zwischen diesen Grenzen liegende Zirkone, welche nach dem Glühen scliwerer werden, sehr oft die obere Grenze von 4,705 erreichend oder wenigstens dieser sehr nahe kommend.

a) Zirkone vom specifischen Gewichte etwa 4. Unter den Mirieralien, welche IDr. Fi. Grünling 7) im Jahre 1897 von Ceylon mitgebracht hat, befinden sich Geschiebe von einem braunen, sehr harten (II. 7,5), glasglïnzenden Minerale, welches nur in dünnen Splittern durchsichtig ist und keine deutliche Spaltbarkeit zeigt. Der Bruch ist splitterig oder muschelig. Jieses Mineral wurde im Laboratorium des Ilerrn Prof. Muthmann analysirt und nach Dessen Mittheilung ergab sich die chemische Zusammenselzung genau als dicjenige des normalen Zirkons, aber das specifische Giewicht entsprach der untersten Grenze, nämlich $4,06$.

Um mich zu überzeugen, ob dieses specifische Gewicht beim Erhitzen constant blieb, bestimmte ich es an denselben Stücken wiederholt vor und nach dem Glühen, und fand:

1) Zeitschr. f. physik. Chemie $4895,16,620 ; 4896,20,314$. Ref. diese Zeitschr. Q9, 173 und 30, 636 .

2. N. Jahrb. f. Min. elc. $1893-96,10$. Beil.-Bd., 470, Diese Zeitschr. 30, 470.

7. Berzelius, Jahresbericht $1848,27,243$.

4) Journal f. prakt. Chemie 1846, 37, 308 .

5) Geolog. Magazine 1875, 2, 332.

6) Dana, system of Mineralogy 1892, 484.

7) Diese Zeitschr. 1900, 33, 210. 


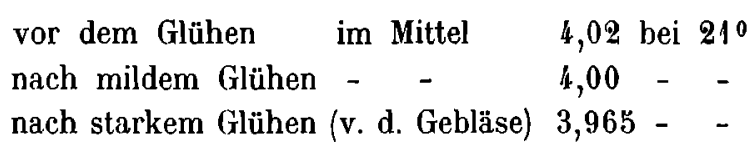

Während des Glühens beobachtete ich eine Farbenveränderung und eine plötzliche Lichtentwickelung. Schon bei dem milden Glühen verliert das Mineral seine braune Farbe und wird zeisiggrün. Diese Farbe behielt es auch nach dem starken Glühen. Das etwas niedriger gewordene specifische Gewicht wie auch das optische Verhalten (siehe unten) künnten für eine Umwandlung sprechen, aber die Stücke sind nach dem Glühen nicht trüb, sondern im Gegentheil durchsichtiger geworden, was dagegen spricht. Es scheint also wahrscheinlicher, dass obige Erscheinung mit den oft bei den Zirkonen beobachteten Phosphorescenzerscheinungen in Beziehung steht. II enneberg, welcher zum ersten Male diese Erscheinung bei den Zirkonen bcobachtete, bemerkt, dass sie sich von der Phosphorescenz anderer Mincralien dadurch unterscheidet, dass sie nur einmal zu beobachten ist und dass sie mit der Verönderung des specifischen Gewichtes und daher mit der Natur des Zirkons selbst in Beziehung steht. G. Spezia) beobachtete ebenfalls diese Erscheinung und meint, dass sie in keiner Beziehung zu den Farbstoffen stche. Auch Scheerer ${ }^{2}$ ) beobachtete sie bei dem Malakon von Hitterö in Norwegen. Ob die Erscheinung nun etwas mit dem Farbstoffe zu thun hat oder vielleicht von dem Gehalte an Argon und IIelium herrührt, welcher in neuerer Zeit von W. Ramsay und M. W. Travers ${ }^{3}$ ) in einigen Malakonen beobachtet wurde, bleibe dahingestellt.

Die optische Untersuchung des Ceyloner Minerals ergab Einaxigkeit mit äusserst schwacher Doppelbrechung positiven Sinnes. An einem von der Firma Voigt und Hochgesang geschliffenen und polirten Prisma vom brechenden Winkel 2.10 18' wurde die Lichtbrechung bestimmt. Wegen der in dickeren Schichten starken Absorption konnte das Prisma nicht orientirt werden, und da die Doppelbrechung so schwach ist, dass die Bilder kaum mit dem Nicol'schen Prisma zu trennen waren, so konnten nur mittlere Einstellungen gemacht werden; diese ergaben die folgenden Werthe des mittleren Brechungsindex, welcher zwar $\omega$ sehr nahe, aber nicht damit identisch ist:

\footnotetext{
1) Diese Zeitschr. 1877, 1, 399.

2) Berzelius, Jahresber. 1846, 25, 327.

3) Royal Soc. 1896, 60, 443; Ref diese Zeitschr. 30, 88.
} 
Die Einaxigkeit bleibt erhalten, und, wie man aus diesen Zahlen ersieht, auch die sehr schwache Doppelbrechung.

Während das angewindte Prisma gleichmässig gefärbt war, zeigten gewisse Stücke Streifensysteme, welche von einem schaligen $\Lambda$ ufbau herrühren.

b) Zirkone von dem specifischen Gewichto otwa 4,\%. Ausser dem von Church beobachteten Zirkon von Mudgee, welcher das spec. Gew. 4,7 hat, sind noch viele Zirkone bekannt, deren specifisches Gewicht der obigen Zahl sehr nahe steht. So fand es A. Schmidt') bei einem australischen Zirkon 4,69:-4,696 und beobachtete an ihm keinc optischen Anomalien. Ich bestimmte das specifische (rewicht bei folgenden Zirkonen: von IRio Doce in Brasilien, welche Ihre Kgl. Hoheit Prinzessin 'Therese von Bayern von ihrer brasilianischen Reise mitgebracht hat, und fand 4,709 bci $21^{0}$. Ihrer Kleinheit halber konnten sie nicht optisch untersucht werden. $\Lambda$ n künstlichen Krystallen, welche $\Lambda$. Haulefeuille und $\Lambda$. Perrey ${ }^{2}$ ) dargestellt hatten, fand ich 4,706 bei $22^{0}$. In seiner Publication sagt llautefeuille, dass diese Stellen mit schwacher Zweiaxigkeit zeigen. Die Krystalle, welche ich zur Verfügung hatte und welche Herr Prof. Groth im Jahre 1900 von Herrn II autefeuille zum Geschenk erhielt, zeigten einige Formen, welche Letzterer nicht beobachtet hat, daher sie wahrscheinlich aus einer späteren Darstellung herrühren. An farblosen Krystallen von der rothen Wand im l'fitschthale, Tirol, fand ich 4,615 bei 210; aber diese Krystalle konnten nicht ohne Gefahr, sie zu zerstüren, von dem Muttergesteine befreit werden, deshalb ist obiger Werth elwas zu klein; A. Gehmacher 3 j hat diesen Zirkon optisch untersucht und gar keine Anomalien beobachtet. An einigen Krystallen von Ceylon, wolche auch von Laterit nicht frei waren, fand ich 4,622. Diese waren ebenfalls optisch nicht anomal. Ius allem Diesen geht hervor, dass die normalen Zirkone das specifische Gewicht von 4,69:-1,709 haben und dass sie keine optischen Anomalien zeigen. Für sie gelten die bisherigen Angaben über die Brechungsindices des Zirkon, nämlich:

$$
\begin{aligned}
& \omega=1,92 \text { für lRoth (Sénarmont), 1,9236-1,9313 für Gelb (Sanger), } \\
& \varepsilon=1,97--\left(\begin{array}{l}
- \\
\varepsilon
\end{array} \quad \text {, 1,9682-1,9931- }-(-)\right. \text {. }
\end{aligned}
$$

Durch die grosse Gefälligkeit des Herrn E. Tornow in Frankfurt a. M. bin ich in der Lage, die genauen Werthe der Brechungsindices mitzutheilen, welche Dieser mittelst eines schönen Prismas vom spec. Gew. 4,654 gefunden hat:

1) Diese Zeitschr. 1891, 19, 56.

2) Compt. rend. 1(00, Ref. diese Zeitschr. 1891, 18, 328.

3) Diese Zeitschr. 1887, 12, 50. 


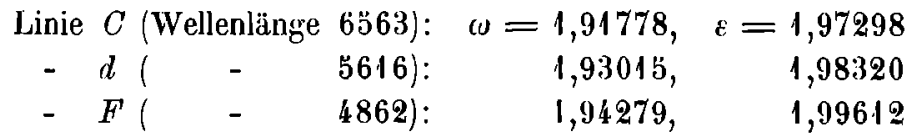

c) Zirkone, deren specifisches Gewicht zwischen den unter a) und b) gegebenen Zahlen liegt. Wie schon erwïhnt, pflegen die Zirkone, deren specifisches Gewicht grösser als 4 und kleiner als 4, $\bar{t}$, dieses durch Glühen zu erhöhen. Es ist merkwürdig, dass wir heutzutage nur einen Versuch zur Erklärung dieser Erscheinung haben; Frank liutley $\left.{ }^{1}\right)$ nämlich erklärt sie durch das Vorhandensein von lilüssigkeits- und Gaseinschlüssen, welche er in der That bei gewissen Zirkonen beobachtet hat. Aber selhstverständlich ist damit nicht diesc Erscheinung bei allen Zirkonen dieser Altheilung zu erklären. Meiner Meinung nach steht diese Erscheinung in der innigsten Bezichung zu den optischen Anomalien, welche zuerst von Mallard ${ }^{2}$, später auch von anderen Mineralogen beobachtet wurde. Der IIauptsache nach wurden die optischen Anomalien an Ceylonischem Zirkon studirt, und wie es scheint immer an derselben Varietiit. So beobachtete Mallard an einer senkrecht zur llauptaxe geschliffenen Platte eines grünlich gefürbten Zirkons einen vollkonmen einaxigen Kern, der von einer gestreiften Ilülle umgehen war; dicse zeigle im convergenten Lichte Lemniscaten von verschiedener Richtung und wechselndem $\Lambda$ xenwinkel. Nach diesen Eigenschaften hielt er den Zirkon für monoklin. Später beobachtete (G. Grattarola ${ }^{\text {) }}$ die Zweiaxigkeit an einem »olivengrünen «, bald an Epidot, bald an Olivin erinnernden Mineral, welches Dr. Beccari von Ceylon mitgebracht hatte; die Analyse zeigte hier cinen Zirkon, welcher aber 2,52 Thonerde und 3,62 Kalk enthielt; er find $2 E=19^{\circ} 44^{\prime} 40^{\prime \prime}$; mit einem Prisma von $35^{\circ} 40^{\prime} 20^{\prime \prime}$ bestimmte er $\gamma=1,9820, \beta=1,9277$, berechnete $\alpha=1,9272 ; 2 V=10^{\circ} 10^{\prime} 28^{\prime \prime}$. Verschieden gefirbte Zirkone hatten auch verschiedene specifische Gewichte, so die hellgrünen $4,05-4,3$, grüne 4,3 $-4,5$, gelbe, rüthliche und farblose $4,69-4,75$.

Meine Beobachtungen sind in Fragmenten von einem grösseren ahgerundeten, ebenfalls grünlichen Krystallstücke von Ceylon ausgeführt, also unbedingt für alle Untersuchungen an dem gleichen Materiale. Das specifische Gewicht ergab sich:

$$
\begin{aligned}
& \text { vor dem Glühen zu } 4,33 \text { bei } 18^{\circ} \text {, } \\
& \text { nach starkem Glühen } 4,66 \text { bei } 20^{\circ} \text {. }
\end{aligned}
$$

Von der schon erwähnten Firma wurde ein Prisma und eine Platte

1) Min. Magaz. and Journal of the miner. Soc., Sept. 1894, Nr. 48; Ref diese Zeitsclır. 27, 107.

2) Annales des mines 1876. 10; Ref. diese Zeitschr. 1. 309.

3) Atli Soc. Tosc. d. Scienc. Natur., Pisa 1879 und 1890 ; Ref. diese Zeitschr. 4, 398 und 23, 170. 
senkrecht zur Hauplaxe geschliffen und polirt. Die Dicke der Platte wurde mit dem Mikrometer zu 2,32:; bestimmt und durch das Mikroskop controlirt. Unter dem Mikroskope war gerade das Umgekehrte von jenem, was Ma]lard beobachtete, zu sehen, nämlich: die Platte war in Partien getheilt. Die Mitte war vollkommen durchsichtig und regelmässig zweiaxig; der Abstand der Axen betrug etwa sechs Theilstriche im Fuess'schen Konoskope, was für die Richtigkeit der obigen von Grattarola gefundenen Zahl spricht. Dieser Kern war von zwei parallelen Streifensystemen umgeben, welche miteinander Winkel von $92^{\circ}$ bildeten. Jiese Partien zeigten das gestürte Bild eines zweiaxigen Minerals. Nach der Formel des Herzog von Chaul. nes ${ }_{c}^{e}-d$ wurde $\alpha=1,8825(d=1,090)$ gefunden. Diese Zahl weicht sehr von 1,9272 ab, welche Grattarola gefunden hat, aber es scheint, dass sein Prisma von einem normalen Zirkon war, weil $c$ und $\beta$ sehr wenig von einander und alle drei schr wenig verschieden von denjenigen des normalen Zirkons sind. Zuletzt wurde die l'latte stark geglüht. Die schwach grünc liarbe verschwand vollkommen. Im Konoskope zeirte jetzt der Kern das Bild eines vollkommen cinaxigen Minerals, ohne irgend welche Spuren von Zweiaxigkeit. An derselben Platte und nach derselben Methode wurde $a=1,9215(d=1,115)$ gefunden; wenn diese Zahlen auch nur auf etwa zwei Decimalen als genau zu betrachten sind, so geht doch daraus unzweifelhaft hervor, dass dies der Brcchungsindex $u$ des normalen Zirkons ist. Die gestreifte Ilülle zeigte ebenfalls das schwarze Kreuz mit den Ringen, aber das Bild war etwas gestört, und die IJurchsichtigkeit der Hülle ist sehr stark becinflusst durch Strcifen, deshalb wurde $\alpha$ nicht bestimmt. Leider war mein Prisma nicht für die Bestimmung der Brechungsindices orientirt, die brechende Kante war zwar parallel mit der Axenebene, aber fiel nicht mit einer Mittellinie zusammen. Trotzdem gehe ich hier die Werthe der lirechungsindices für $N a$-Licht, welche ich gefunden habe, nämlich:

Schwing. parallel d. brech. Kante: 1,8870

Schwing. senkrecht z. brech. Kante: 1,9115

Da die Kahl 1,8870 dem $\alpha$ entspricht, aber etwas grösser scin muss, so scheint der Werth 1,8825 richtig zu sein. Erste Mittellinie ist c.

Aus allem, was über diesen Zirkon gesugt ist, folgt: 1)ie Krystalle zcigen Scctorentheilung, der Kern ist entweder optisch vollkommen einaxig, wie Mallard beobachtet hat, und die Hülle anomal, oder umgekehrt: der Kern ist zweiaxig und die llülle anomal, wie an meinem Beispiele beobachtet wurde. Es ist ganz klir, dass im ersten Falle zuerst ein optisch einaxigres Mineral zu Stande gekommen ist und dass beim Fortwachsen veränderte Umstände eingetreten sind, welche den Anlass zur Entstehung eines zweiaxigen Ninerals gegeben haben. Im zweiten Falle war es umgekehrt, 
zuerst ist cin zwciaxiges Nineral (nach dem $A x$ enbilde rhombischj entstanden und dieses unter veränderten Bedingungen in eine einaxige Modification übergetreten. Auch der Winkel 920, unter welchem sich die Streifensysteme schneiden, spricht dafür, dass hier zuerst ein zweiaxiges Mineral vorhanden war. Wir haben also hier eine metastabile Modification von kleinerem specifischem Gewichte. Die optischen Anomalien zeigen uns, dass sich die Bedingungen während seiner Entstẹhung änderten.

Die Zweiaxigkeit an den Zirkonen hat ferner noch A. Madelung 1) beobachtet und gefunden: Slatoust (Ural), schwach aber deutlich zweiaxig; dunkel röthlicher Zirkon von Ceylon deutlich zweiaxig, und hell röthlicher, ebenfalls von Ceylon, zeigten abwechselnd auftretende Verdunkelung.

Während des Glühens beobachtete ich auch bei diesem Zirkon die Farbenänderung, aber keine Phosphorescenzerscheinungen; das Mineral wird vollkommen farblos. Mir ist nicht gelungen, den einmal schon entfürbten Zirkonen wieder durch Reduction die Farbe zu geben, was sonst Niemandem ausser Spezia geglückt ist.

Fassen wir das Gesagtc über die drei Zirkonvarietäten zusammen, so dürfte daraus hervorgehen, dass es sich bei c) um eine zweiaxige Modification vom spec. Gew. $4,3 \mathrm{ca}$. handelt, welche durch Glühen in den normalen Zirkon b) vom spec. Gew. 4,7 ca. umgewandelt wird. Völlig verschieden verhält sich das Mineral vom spec. Gew. 4,0, eine Erscheinung, welche zu der Vermuthung führt, dass dasselbe an Stelle des Zirkoniums ein anderes, demselben sehr nahestehendes Element enthält.

Krystallographische Notizen über normalen Zirkon. Die erste genaue Messung an dem Zirkon hat W. Haidinger i. J. 1817 ausgeführt und für den Grundwinkel $(111):(1 \overline{1} 1)$ den Werth $36^{\circ} \mathbf{1}^{\prime}$ gefunden. Spälere Messungen von Kupf(er $\left.{ }^{2}\right)$, Ko kscharow ${ }^{3}$ ) und Dauber $\left.{ }^{4}\right)$ unterscheiden sich sehr wenig von Obigem, so dass Dauber, welcher mehrere Krystalle von verschiedenen Lucalitäten gemessen hat, als Mittelwerth für den Grundwinkel $360^{\circ} 40^{\prime} 3 "$ gah, welcher auch heutzutage angenommen wird, obwohl er nicht für alle Zirkone gilt. So z. B. fand N. v. Kokscharow jun. ${ }^{5}$ ) diesen Winkel an Zirkonen aus den südamerikanischen Seifen $56^{\circ} 45^{\prime}$ im Mittel sorgfältiger Messungen.

Ich habe Krystalle von folgenden in der Münchener Sammlung befindlichen Localitäten gemessen:

Rio Doce in Brasilien (vielleicht identisch mit denen, welche

1) Mitgetheilt von A. Schrauf, diese Zeitschr. 1883, 7, 75.

2) Preisschrift 66.

3) Material. z. Miner. Russlands $1858,3,168$.

4) Pogg. Ann. 1859, 57, 275.

3) Material. z. Min. Russl. 10, 23. 
H. Gorceix ${ }^{1}$ ) beschrieben hat und welche vom Rio Matipo, einem Nebenflusse des Rio Doce, stammen). Kleine, farblose oder röthliche Krystalle, manchmal von ausgezeichneter Ausbildung, mit folgenden Formen: $\{100\}$, $\{110\},\{111\},\{311\},\{101\}$ und wahrscheinlich $\{411\}$, welche vom Rath einmal an Krystallen vom Pfitschthale beobachtet hat. Die Krystalle sind kurzprismatisch. Gewöhnlich sind beide Prismen $\{110\}$ und $\{100\}$ vorhanden mit $\{111\}$ und $\{311\}$, aber häufig nur $\{100\}$ und $\{311\}$. Die Form $\{101\}$ ist gar nicht selten.

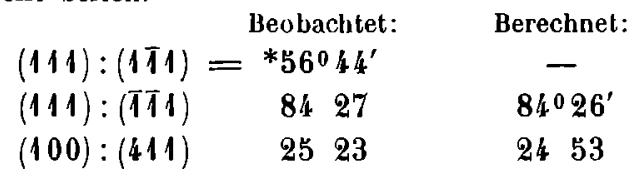

Nord-Carolina. Den obigen sehr ähnliche Combination: $\{100\},\{110\}$, $\{111\},\{311\},\{101\},\{331\},\{511\}$ und die neuen Formen $\{401\}$ und $\{535\}$.

$\begin{array}{lcc}(111):(1 T 1)= & \begin{array}{c}\text { Beobachtet: } \\ * 56^{0} 44^{\prime}\end{array} & \text { Berechnet: } \\ (111):(1 T 1) & 8424 & 84026^{\prime} \\ (100):(401) & 2040 & 2121 \\ (311):(401) & 1749 & 1734 \\ (101):(535) & 1742 & 1737 \text { [Zone }(111):(101)]\end{array}$

Rothe Wand im Pfitschthal. Zuersl von vom Rath $\left.{ }^{2}\right)$ gemessen, welcher an ihnen die Combination $\{100\},\{110\},\{111\},\{311\},\{331\},\{511\}$, und $\{111\}$ beobachtet hat. Später wurden diese Krystalle von Dauber und Gehmacher gemessen. Diese haben die Form \{111\} nicht beobachtet. Ausser obigen beobachtete ich $\{301\},\{881\},\{991\}$ und wahrscheinlich $\{544\}$ und $\{655\}$, welche bis jetzt an den Zirkonen nicht beobachtel waren.

$\left.\begin{array}{lrc} & \begin{array}{c}\text { Beobachtet: } \\ (111):(1 T 1)=\end{array}{ }^{*} 56^{0} 37^{\prime} & \text { Berechnel: } \\ (111):(T 11) & 8418 & 84014^{\prime} \\ (100):(301) & 2729 & 2730 \\ (311):(301) & 1633 & 1629 \\ (110):(881) & 734 & 754 \\ (100):(991) & 649 & 656 \\ (111):(635) & 430 & 436 \\ (111):(544) & 630 & 538\end{array}\right)$ Zone $(311):(111)$

Künstliche Krystalle, von Hautefeuille dargestellt. Den vorigen

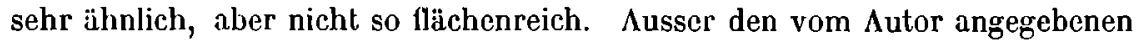
Formen $\{110\},\{111\}$ und $\{101\}$ beobachtete ich noch $\{311\}$.

4! Compt. rend. 1884, 98, 1446; Ref. diese Zeitsebr. 11, 639.

2) Pogg. Anual. 108, 3:3. 


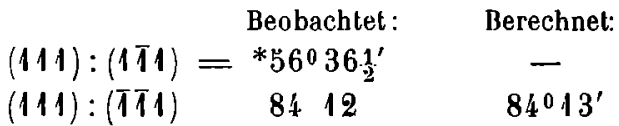

Wie man sieht, variiren die Werthe für den Grundwinkel, und zwar nicht zufällig, weil die berechneten Werthe besser mit den beobachteten, als init jenem von Dauber übereinstimmen. Wenn wir aber von diesen vier Werthen das Mittel nehmen, so erhalten wir $56^{\circ} 40^{\prime} 30^{\prime \prime}$, was von dem von Dauber sehr wenig abweicht.

Die neuen Formen, ausser $\{301\}$, sind sehr selten, manche nur einmal beobachtet, und sehr schmal, gewöhnlich dort vorhanden, wo der Krystall von gestörter Bildung war. Nur $\{301\}$ ist dreimal gesehen worden, es ist glatt und gab gute Reflexe.

Künstliche Irystalle von Molybdändioxyd. Im J. 1881 haben F. Mauro und 1R. Panebianco ${ }^{1}$ ) krystallisirtes Molybdändioxyd dargestellt, indem sie Kaliumcarbonat mit den Anhydriden von Molybdän- und Borsäure in dem Verhältnisse gleicher Moleküle $\mathrm{K}_{2} \mathrm{O}, \mathrm{MoO}_{3}$ und $\mathrm{B}_{2} \mathrm{O}_{3}$ schmolzen. Die undurchsichtigen, metallisch glänzenden Krystalle, von kupferrother bis bleigrauer Farbe, waren tetragonal; $a: c=1: 0,5774$. Gewöhnliche Combination: $\{110\},\{101\},\{221\}$ und $\{211\}$; seltener kommen noch dazu die Formen $\{001\},\{$ ơl 0$\},\{201\},\{301\},\{401\},\{111\},\{332\},\{132\},\{532\}$ und \{411\}. Das spec. Gew. 6,14. Die Autoren bemerken, dass diese Krystalle Analogie mit den Mineralien der Zinnerzgruppe haben.

In neuerer Zeit erhielt Herr Prof. Muthmann bei der Elektrolyse von molybdänsaurem Kalium Krystalle von Molybdändioxyd. Dieselben stimmen in Farbe und Glanz vollkommen mit obigen überein. Sie zeigten kupferrothe Farbe auf den Domen- und bleigraue auf den Prismenflächen. Aber der Habitus der Krystalle, die Zwillingsbildung, sowie auch die Winkelwerthe sprechen für monokline, obwohl der tetragonalen sehr nahe stchende Symmetrie. Combination: $q\{011\}, r\{101\}, m\{110\}$ und selten noch $x\{121\}$ und $o\{(11\}$. Um die Aehnlichkeit der Winkel besser zu veranschaulichen, stelle ich die beiden Modificationen ncben einander.

Monokline Mod.

$$
a: b: c=0,9869: 1: 0,5765, \beta=91034^{\prime}
$$

Beobachtet:

$q: q=(011):(0 \overline{1} 1)={ }^{*} 59^{0} 55^{\prime} \pm 2^{\prime}$

$q: r=(011):(101)$

$r: m=(101):(110)$

$m: m=(110):(1 \overline{1} 0)$

$m: q=\left\{\begin{array}{l}(110):(011) \\ (110):(011)\end{array}\right.$
Berechnet:

*41 $201 \pm 19$

*68 $16 \pm 2$

$8922= \pm 21$

$6831 \pm 50$

70) 24
T'etragonale Mod. $a: a: c=1: 1: 0,5774$.

Berechnet:

$60^{\circ} 0^{\prime}$

425

6918

$90 \quad 0$

$69 \quad 18$

6918

1) Accarl. a. Linc. Memorie 1881. 9, 5 griugno; Ref. diese Zeilscha. 1882. (i, $28:$;. 


\begin{tabular}{|c|c|c|c|}
\hline$m: o=(T 10$ & $\begin{array}{l}\text { Beobachtet: } \\
=51019^{\prime}\end{array}$ & $\begin{array}{c}\text { Berecbnet: } \\
51026^{\prime}\end{array}$ & $\begin{array}{c}\text { Berechnet: } \\
50^{\circ} 46^{\prime}\end{array}$ \\
\hline$o: o=(\bar{T} 11):(\bar{T} \overline{1} 1)$ & 5333 & $53 \quad 34$ & 538 \\
\hline$p: x=(011):(121)$ & $2830 \pm 25$ & 280 & - \\
\hline
\end{tabular}

Die Krystalle sind klein, gestreckt und niemals beiderseits entwickelt, wodurch die Bestimmung sehr erschwert wird. Um müglichst genaue Werthe $\mathrm{zu}$ el'zielen, wurden etwa $30 \mathrm{Krystalle} \mathrm{gemessen.} \mathrm{Einfache} \mathrm{Krystalle} \mathrm{(Fig.} \mathrm{5)}$ zeigen gewöhnlich $q$ und $r$ im Gleichgewicht entwickelt und oft sehr schmale Prismenflächen. Es wurden auch mehrere Zwillinge beobachtet, von denen einer vollstiindig entwickelt war (Fig. 6a); er zeigte am oberen Pole einen einspringenden Winkel $q: q=$ $42046^{\prime}$, die Prismenflächen fallen

Fig. 5.

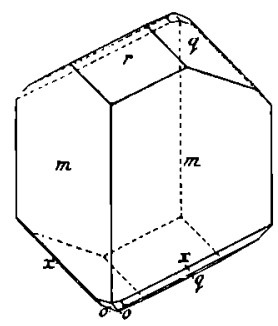

Fig. 6 a und $6 \mathrm{~b}$.

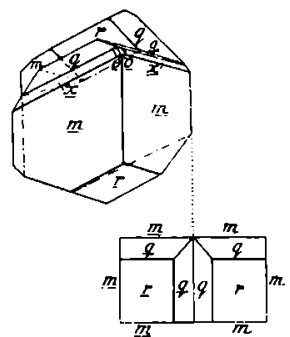
fast zusammen und ain unteren Pole (Fig. 6b) erscheint eine fast rhombische Pyramide; das weist darauf hin, dass $m(110) \mathrm{Zwillingsebene}$ ist und dass wir am unteren Pole $q$ und $q$, und $r$ und $r$ hıben; die beiden anderen $q$-Flächen sind durch dic Verwachsung verschwunden. I)ie zurückgeblicbenen lïlichen bilden mit einander die Winkel:

Beobachtet:

$$
\begin{aligned}
& q: r=(011):(101)=41025 \\
& r: r=(101):(101) \\
& q: q=(011):(011)
\end{aligned}
$$

Berechnet:

$41020^{\prime}$

4.328

4.258

Sind die Krystalle nicht beiderseits entwickelt, was gewühnlich der Fall ist, so ist bei diesen, manchmal schr schlechten Krystallen die Zwillingsbildung seh" schwer zu erkennen.

Jas spec. Gew. fand ich 6,342 bei $1.4^{\circ} \mathrm{C}$, also auch demjenigen der tetragonalen Modilication schr nahc.

\section{Die topischen Axen bei den Mineralien der Zinmerzgruppe.}

Um zu schen, in welcher Beziehung das Molybdändioxyd zu den Mineralien der Zinnerzgruppe und diese zu cinander stehen, wurden die topischen $\Lambda$ xen für alle Glieder dieser Gruppe ausgerechnet. Da aber das spec. Gew. des Zinnerzes in ziemlich weiten Grenzen angegelen ist, nïmlich von 6,3 -6,8433, so bestimmte ich es noch cimmal an künstlichen Krystallen, welche die Firma Krantz in Bonn lieferte und welche $\Lambda$. Arzruni beschricben hat $\left.{ }^{1}\right)$. Ich fand 6,992-..7,044 bei 16\%. Diese Kryställehen sind farblos oder violell

Fig. 7.

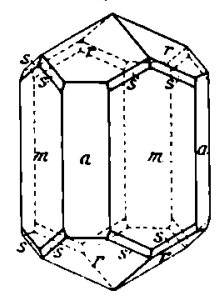

1. Dicse Zeitschr. 18!6, 25. 466. 
gefärbt, manchmal von ausgezeichneter Ausbildung. Einige wurden gemessen und zeigten die Combination: $a\{100\}, m\{110\}, r\{101\}, s\{431\}$ und $T\{110\}$. Da die beobachtcten und berechneten Werthe sehr kleine Unterschiede zeigen, so gebe ich hicr die Winkeltabelle.

Beobachtet:

Berechnet.

Stevanović: Arzruni:

$\begin{array}{lccc}r: r=(101):(101) & * 67051^{\prime} & - & 67048^{\prime} \\ r: m=(101):(110) & 6645 & 66^{0} 45 s_{2}^{\prime} & 66451 \\ r: r=(101):(011) & 4629 & 1628 \frac{1}{2} & 4630 \\ s: s=(431):(4 \overline{3} 1) & 719 & 714 & - \\ s: m=(431):(110) & 1825 & 1825 & - \\ s: s=(431):(341) & 1515 & 1632 & - \\ T: a=(410):(100) & 140-16^{0} \text { appr. } & 142 & -\end{array}$

Das aus obigen Zahlen berechnete Axenverhältniss ist $a: c=1: 0,67259$. Die Formen $s$ und $T$ sind am Zinnerz zum ersten Male beobachtet, sie sind schmal, deshalb die Indices für $T$ unsicher (sie künnen auch $\{720\}$ sein).

Stellen wir jetzt, nach ihren Molekulargewichten angeordnet, die Mineralien der Zinnerzgruppe nebst dem tetragonalen Molybdändioxyd zusammen, so haben wir:

\begin{tabular}{|c|c|c|c|c|c|c|}
\hline & $M$ & $d$ & $V$ & $a: c$ & $x$ & $\omega$ \\
\hline Rutil $\mathrm{TiO}_{2}$ & 80,1 & 4,25 & $18,8,17$ & $1: 0,6441$ & 3,081 & 1,984 \\
\hline Polianit $\mathrm{MnO}_{2}$ & 87,0 & 5,04 & 17,262 & $1: 0,6647$ & 2,960 & 1,969 \\
\hline Zirkon ${ }_{2}^{\operatorname{ZrSi}} \mathrm{O}_{2}$ & 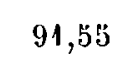 & 70 & & $1 \cdot 0$ & & 1,999 \\
\hline Molybdändiox. $\mathrm{MoO}_{2}$ & & 6,4 & & & & \\
\hline Zinnerz $\mathrm{SnO}_{2}$ & 150,00 & 7,018 & 21,4 & $1: 0,6726$ & 3,608 & 2,136 \\
\hline Thorit $\frac{T h}{2}-O_{2}$ & +70 & 5,40 & 00,000 & $1: 0,6402$ & 76 & 2,310 \\
\hline lattnerit $\mathrm{PbO}_{2}$ & 38,9 & 8,5 & 28,106 & $1: 0,6764$ & 3,468 & $2,3 / 45$ \\
\hline
\end{tabular}

Aus dieser Tabelle ersieht man, dass von den Mineralien dieser Gruppe, deren Isomorphismus noch nicht angezweifelt ist, nämlich: Rutil, Polianit, Zinnerz und Plattnerit, das erste, dritte und vierte eine Regelmässigkeit zeigen, nämlich mit dem Steigen des Molekulargewichtes ein Steigen auch aller anderer Functionen, wie Volumina, Axenverhältniss, $\chi$ und cu, während Polianit abweicht. Bei dem Zirkon und Thorit für sich genommen scheint mit dem Steigen des Molekulargewichtes ein Sinken des Axenverhältnisses stattzufinden; die anderen Functionen steigen. Polianit und Molybdïndioxyd zeigen gar keine Regelmïssigkeit.

Ich benutze diese Gelegenheit, meinem geehrten Lehrer, Herrn Prof. P. v. Groth, für Alles, was er für meine mineralogische Ausbildung gethan hat, meinen besten Dank auszusprechen. Elsenso bin ich dem Ierrn Prof. Muthmann für freundliche Mittheilungen zu grossem Danke verpflichtet. 